A pidologie, 1982, 13 (2), 157-168.

\title{
ÉVOLUTION DE LA CONSANGUINITÉ DANS UNE POPULATION D'ABEILLES
}

\author{
CHEVALET $\mathrm{Cl}$. \\ I.N.R.A., Laboratoire de Génétique Ceilulaire, 31320 Castanet Tolosan \\ CORNUET J.-M. \\ I.N.R.A., Station de Zoologie, 84140 Montfavet
}

\begin{abstract}
RÉSUMÉ
Pour une population d'abeilles, caractérisée par les nombres de reines produisant respectivement les reines et les mâles de la génération suivante, et par le taux d'immigration, on détermine la loi de l'évolution du taux moyen de consanguinité. Pour une population ouverte, on calcule la valeur limite du coefficient de consanguinité et l'on donne des abaques de cette quantité; pour une population fermée, on donne l'expression de l'effectif génétique.
\end{abstract}

\section{INTRODUCTION}

Lorsqu'on effectue une sélection à l'intérieur d'une population, l'augmentation de la consanguinité est inévitable (Crow et KIMURA, 1970) du fait de la réduction des effectifs engendrée par le choix d'un nombre limité de reproducteurs.

Chez l'abeille, la consanguinité se révèle néfaste à deux niveaux. D'une part, elle entraine une augmentation de la probabilité de fécondation entre gamètes portant le même allèle sexuel (WoYKe, 1972), ce qui conduit à une baisse de la viabilité du couvain et représente une première cause de diminution de la productivité des colonies. D'autre part, le comportement des ouvrières est modifié (PLASs, 1953) : déficience de l'élevage, baisse de l'activité de nettoyage entrainant une sensibilité accrue aux maladies parasitaires. Pour ne pas voir le progrès dû à la sélection contrebalancé par les effets néfastes de la consanguinité, il est nécessaire de tenir compte de ce facteur lors de la conception d'un plan de sélection. 
Dans ce travail, nous nous sommes placés dans le cadre d'un plan, détaillé ailleurs (CoRnuet et Chevalet, 1981), de sélection combinée de reines avec fécondation naturelle par des mâles provenant en partie de reines sélectionnées.

L'étude a porté sur l'évolution des coefficients de consanguinité et de parenté moyens définis par MALECOT (1948) au cours des générations successives de la population soumise à la sélection. Ces coefficients sont définis pour un locus indépendant du locus sexuel et des locus contribuant à l'expression des caractères sélectionnés : seule l'incidence de la sélection sur les effectifs des animaux reproducteurs est donc prise en compte dans le calcul.

\section{I. - RAISONNEMENT}

\section{1. - Description succincte du plan de sélection}

A chaque génération, un nombre fixe $R$ de reines est sélectionné d'après la valeur de leur propre colonie et de colonies ayant à leur tête des reines qui leur sont sœurs. Chacune de ces $\mathbf{R}$ reines donne naissance à $\mathbf{S}$ filles. Ces RS reines filles sont fécondées naturellement et on suppose qu'en moyenne une proportion $\beta(0 \leqslant \beta \leqslant 1)$ des mâles qui les inséminent proviennent des $P$ meilleures reines sélectionnées $(P \leqslant R)$. Ces RS reines sont testées et parmi elles seront sélectionnées $R$ nouvelles reines qui seront les mères de la génération suivante de reines.

\section{2. - Établissement des relations de récurrence}

Selon la définition de MALECOT (1948), le coefficient de consanguinité $\mathrm{F}_{a}$ d'un individu diploïde $a$ représente la probabilité qu'à un locus donné, les deux allèles portés par $a$ soient identiques par descendance, c'est-à-dire soient la copie d'un même allèle ancestral. Ce coefficient n'a de sens chez l'abeille que chez les femelles puisque les mâles sont haploïdes. Par contre, le coefficient de parenté $\Phi_{a b}$ entre les individus $a$ et $b$, défini comme la probabilité qu'à un locus donné, un allèle tiré au hasard chez $a$ soit identique par descendance à un allèle tiré au hasard chez $b$ ne pose pas de problème particulier d'application chez l'abeille, si ce n'est que le mâle étant haploïde, nous avons la relation évidente :

$$
\Phi_{\tilde{a} b}^{\tilde{b}}=\Phi_{m b}
$$

où $m$ est la mère du mâle $\tilde{a}$, et $b$, une femelle ou un mâle.

Dans le raisonnement qui suit, nous allons rechercher les relations qui lient les coefficients de consanguinité et de parenté d'une génération avec ceux des générations précédentes. Pour cela nous ne considérerons que les individus sexués, puisque les coefficients de consanguinité des ouvrières sont équivalents à ceux des reines de la même gèneration (elles ont les mêmes parents en moyenne) et que leur coefficient de parenté ne présente pas d'intérêt puisqu'elles ne peuvent avoir de descendance (on néglige ici volontairement les mâles issus d'ouvrières). 
Considérons tout d'abord une reine $a$ de la génération $g$ et recherchons quelle relation lie son coefficient de consanguinité $F_{a}$ aux coefficients des générations précédentes. Sa généalogie est représentée à la figure 1. Le coefficient de consanguinité de $a$ est égal, par définition au coefficient de parenté entre sa mère $b$ et son père $\tilde{c}$.

$$
F_{a}=\Phi_{b c}
$$

Ou bien $\tilde{c}$ ne provient pas d'une reine sélectionnée (Probabilité $=1-\beta$ ) et nous considérerons alors que la parenté avec $a$ est nulle; ou bien il provient d'une reine sélectionnée $p$ et dans ce cas, $\Phi_{b c}=\Phi_{b p}$. Nous avons donc la relation :

$$
\mathrm{F}_{a}=(1-\beta) 0+\beta \Phi_{b p}=\beta \Phi_{b p}
$$

L'individu $a$ n'étant pas davantage précisé, on peut le considérer comme tiré au hasard dans sa génération $g$. Il nous reste donc à calculer la valeur moyenne espérée de $\Phi_{b p}$ ainsi défini.

Deux cas incompatibles se présentent :

- ou bien $b$ est fille de $p\left(\right.$ probabilité $\left.=\frac{1}{\mathrm{R}}\right)$, ce qui revient à assimiler $p$ à $d$ et $\Phi_{b p}$ à $\Phi_{b d}$ (fig. 1). Or,

$$
\Phi_{b d}=\frac{1}{2} \Phi_{e d}^{\sim}+\frac{1}{2} \frac{1+F_{d}}{2}
$$

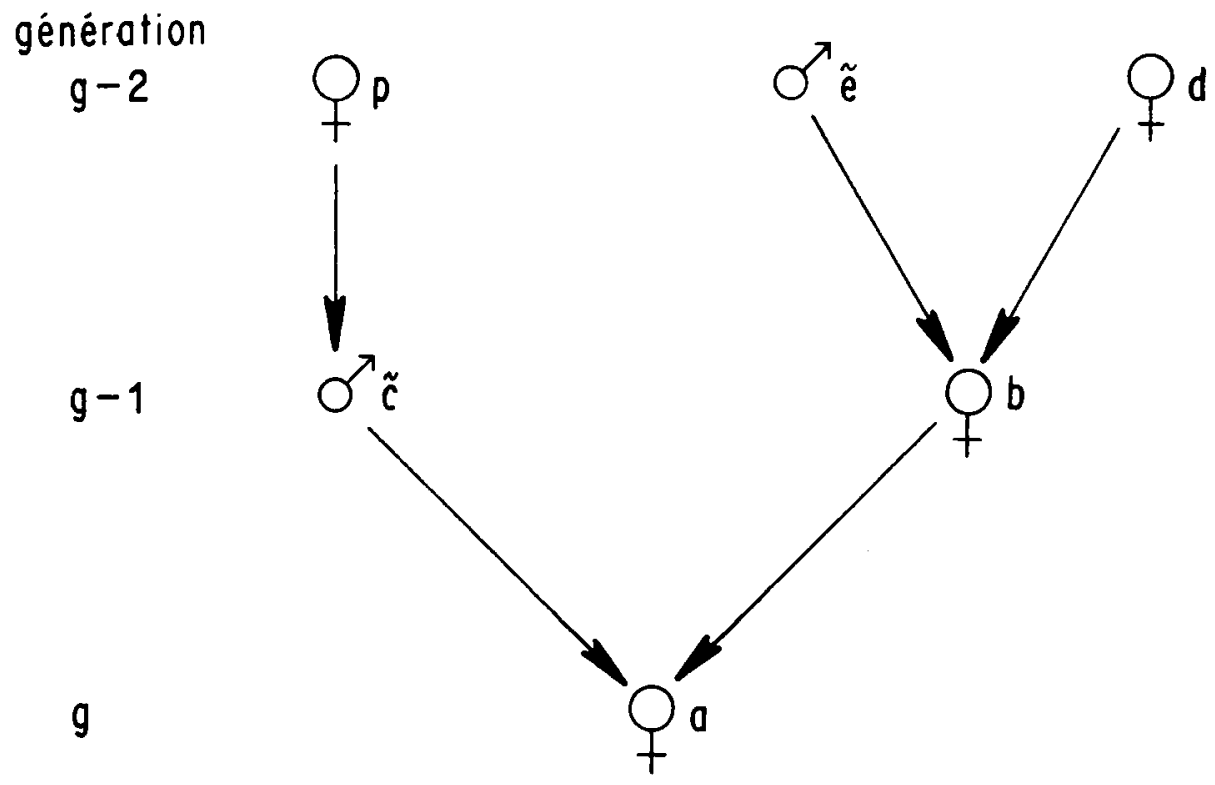

FIG. 1. - Généalogie d'une reine (a) pour calculer l'évolution $d u$ coefficient de consanguinité.

FIG. 1. - Relationship design of a queen (a) to compute the change with time of the consanguinity coefficient. 
En effet, l'allèle tiré chez $b$ a une chance sur deux de provenir de son père $\tilde{e}$ et il sera identique à l'allèle tiré chez $d$ avec la probabilité $\Phi_{e d}^{-}$. Ou bien il provient de $d$ elle-même et dans ce cas, ou bien il est identique à l'allèle tiré chez $d$ (probabilite $\frac{1}{2}$ ) ou bien il est identique à l'autre allèle de $d\left(\right.$ probabilitè $\left.\frac{1}{2}\right)$ qui sera identique à l'allèle tiré avec la probabilité $F_{d}$.

Mais $\Phi_{c d}^{\sim}$ n'est autre que $\mathrm{F}_{b}$, coefficient de consanguinité d'une reine de la génération $g-l$ et $\mathrm{F}_{d}$ est le coefficient de consanguinité d'une reine de la génération $g-2$;

- ou bien $b$ n'est pas la fille de $p\left(\right.$ probabilité $\left.=1-\frac{1}{\mathrm{R}}\right)$. Dans ce cas :

$$
\Phi_{b p}=\frac{1}{2} \Phi_{\bar{e} \rho}+\frac{1}{2} \Phi_{d p}
$$

$\Phi_{\grave{e} p}$ est en moyenne égal à $\Phi_{\grave{e} d}=\mathrm{F}_{b}$.

$\Phi_{d p}$ est un nouveau type de coefficient : coefficient de parenté entre deux reines distinctes $d$ et $p$ de la génération $g-2$.

En notant :

$\mathrm{F}_{g} \quad$ le coefficient de consanguinité moyen d'une reine de la génération $g$ (par exemple $a$ );

$\mathrm{F}_{g-1}$ le coefficient de consanguinité moyen d'une reine de la génération $g-I$ (par exemple $b$ );

$\mathrm{F}_{\boldsymbol{g}^{-2}}$ le coefficient de consanguinité moyen d'une reine de la génération $g-2$ (par exemple $d$ ), et

$\Phi_{g-2}$ le coefficient de parenté moyen entre deux reines distinctes de la génération $g-2$ (par exemple $p$ et $d$ );

l'équation $\mathrm{F}_{a}=\beta \Phi_{p b}$ devient :

$$
\mathrm{F}_{\mathrm{g}}=\beta\left(\frac{1}{2} \mathrm{~F}_{\mathrm{g}-1}+\frac{1}{2 \mathrm{R}} \frac{1+\mathrm{F}_{\mathrm{g}-2}}{2}+\frac{1}{2}\right) 1-\frac{1}{\mathrm{R}}\left(\Phi_{\mathrm{g}-2}\right)
$$

L'apparition du coefficient $\Phi_{g-2}$ dans l'équation nécessite l'établissement d'une équation du même type pour le coefficient $\Phi_{g}$, qui est représenté par $\Phi_{a a^{\prime}}$ (fig. 2). Il y a quatre provenances équiprobables pour les allèles tirés au hasard chez $a$ et $a^{\prime}$. D'où :

$$
\Phi_{a a^{\prime}}=\frac{1}{4}\left(\Phi_{\tilde{c} \tilde{c}^{\prime}}+\Phi_{\bar{c} b^{\prime}}+\Phi_{b \tilde{c}^{\prime}}+\Phi_{b b^{\prime}}\right)
$$

Réglons, tout d'abord, le cas de $\Phi_{\tilde{c} b^{\prime}}$ et $\Phi_{b \tilde{c}^{\prime}}$ équivalents en moyenne à $\Phi_{\tilde{c} b}$ ou $\Phi_{\tilde{c}^{\prime} b^{\prime}}$, c'est-à-dire $\mathrm{F}_{g^{\text {. }}}$. Considérons ensuite $\Phi_{b b^{\prime}}$ qui prend deux valeurs possibles selon que $b$ et $b^{\prime}$ sont confondues ou non. La probabilité qu'il s'agisse de la même reine est égale à $\frac{1}{\mathrm{R}}$ et dans ce cas $\Phi_{b b^{\prime}}$ prend la valeur $\frac{1+\mathrm{F}_{g-1}}{2}$. Dans le cas contraire 

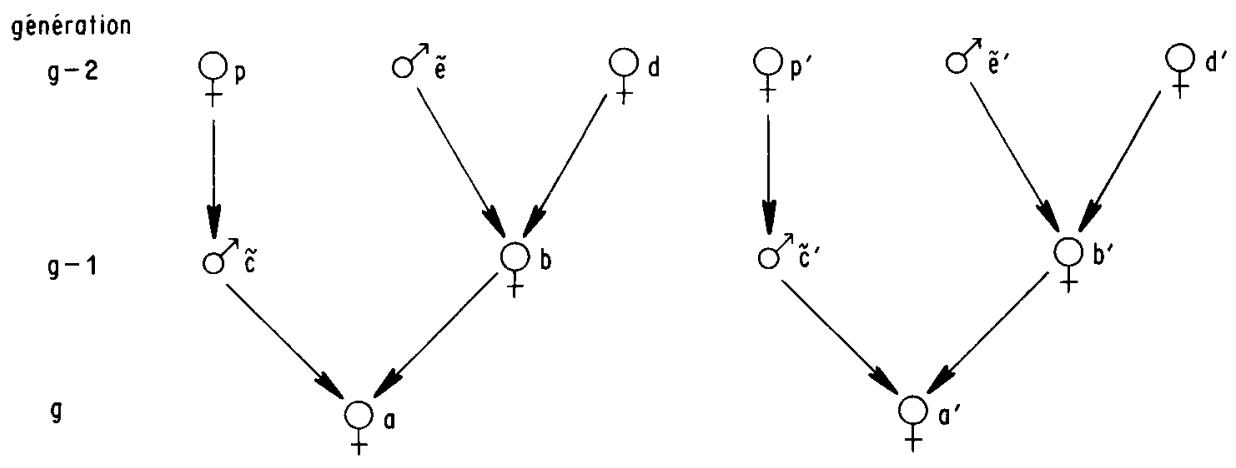

FIG. 2. - Généalogie de deux reines (a) et ( $\left.a^{\prime}\right)$ pour calculer l'évolution du coefficient de parenté.

FIG. 2. - Relationship design of two queens $(a)$ and $\left(a^{\prime}\right)$ to compute the change with time of the kinship coefficient.

probabilitè $=1-\frac{1}{\mathrm{R}} \quad, \Phi_{b b^{\prime}}$ est tout simplement égal à $\Phi_{g^{-1}}$ selon la définition cidessus. D'où :

$$
\Phi_{b b^{\prime}}=\frac{1}{\mathbf{R}} \frac{1+\mathbf{F}_{g-1}}{2}+\left(1-\frac{1}{\mathbf{R}}\right) \Phi_{g-1}
$$

Enfin, il reste $\Phi_{\tilde{c} \tilde{c}^{\prime}}$. L'identité entre $\tilde{c}$ et $\tilde{c}^{\prime}$ n'est possible que si $b$ et $b^{\prime}$ sont confondues, puisqu'un mâle ne peut s'accoupler qu'à une seule reine.

Il y a donc deux cas à prendre en compte :

- Les reines $b$ et $b^{\prime}$ sont confondues (probabilité $\frac{1}{\mathrm{R}}$ ). Dans ce cas, il faut à nouveau considérer deux éventualités incompatibles :

- les deux mâles sont identiques avec la probabilité $1 / f$ selon les notations de Chevalet et Cornuet (1981) et l'identité des gènes est certaine;

- les deux mâles sont différents (probabilité $1-1 / f$ ), ils proviennent tous deux de reines sélectionnées (probabilité $\beta^{2}$ ) et les gènes qu'ils portent sont identiques avec la probabilité $\Phi_{p p^{\prime}}$

- Les reines $b$ et $b^{\prime}$ sont distinctes. Alors la seule possibilité d'identité des gènes de $\tilde{c}$ et $\tilde{c}^{\prime}$ provient de l'apparentement de leurs mères $p$ et $p^{\prime}$ pour peu que ces dernières soient des reines sélectionnées (probabilité $\beta^{2}$ ).

D'où $\Phi_{\tilde{c} \bar{c}^{\prime}}$ s'écrit :

$$
\Phi_{\tilde{c} \tilde{c}^{\prime}}=\frac{1}{\mathrm{R}}\left[\frac{1}{f}+\left(1-\frac{1}{f}\right) \beta^{2} \Phi_{p p^{\prime}}\right]+\left(1-\frac{1}{\mathrm{R}}\right) \beta^{2} \Phi_{p p^{\prime}}
$$


Par un raisonnement analogue à celui que nous venons de faire pour $\Phi_{b b^{\prime}}$, nous obtenons la relation :

$$
\Phi_{p p^{\prime}}=\frac{1}{\mathrm{P}} \frac{1+\mathrm{F}_{g-2}}{2}+\left(1-\frac{1}{\mathrm{P}}\right) \Phi_{g-2}
$$

Si l'on remplace $\Phi_{a a^{\prime}}$ par $\Phi_{g}$, on obtient la deuxième équation de récurrence :

$$
\begin{gathered}
\Phi_{g}=\frac{1}{2} \mathrm{~F}_{g}+\frac{1}{4} \beta^{2} \\
\left(1-\frac{1}{\mathrm{R} f}\right)\left[\frac{1}{\mathrm{P}} \frac{1+\mathrm{F}_{g-2}}{2}+\left(1-\frac{1}{\mathrm{P}}\right) \Phi_{g-2}\right]+\frac{1}{4 \mathrm{R} f} \\
+\frac{1}{4}\left[\frac{1}{\mathrm{R}} \frac{1+\mathrm{F}_{g-1}}{2}+\left(1-\frac{1}{\mathrm{R}}\right) \Phi_{g-1}\right]
\end{gathered}
$$

\section{3. - Résolution des équations de récurrence}

Les équations (1) et (2) constituent un système complet entre $\mathrm{F}_{g}$ et $\Phi_{g}$ portant sur 3 générations successives $(g, g-1, g-2)$. La résolution de ce système n'apparaît pas de façon évidente. Aussi nous proposons de transformer ces deux équations en quatre équations, pour obtenir un système matriciel du type $\mathrm{X}_{\mathrm{g}}=\mathrm{A} \mathrm{X}_{\mathrm{g}-1}+\mathrm{B}$.

Pour cela, nous posons :

$$
\mathrm{C}_{\mathrm{g}}=\frac{1}{\mathrm{R}} \frac{1+\mathrm{F}_{\mathrm{g}-1}}{2}+\left(1-\frac{1}{\mathrm{R}}\right) \Phi_{\mathrm{g}-1}
$$

puisque cette expression apparaît dans les deux équations (1) et (2). Alors (1) devient :

$$
\mathrm{F}_{\mathrm{g}}=\beta\left(\frac{1}{2} \mathrm{~F}_{\mathrm{g}-1}+\frac{1}{2} \mathrm{C}_{\mathrm{g}-1}\right)
$$

De même, nous posons :

$$
\mathrm{D}_{\mathrm{g}}=\beta^{2}\left[\frac{1}{\mathrm{P}} \frac{1+\mathrm{F}_{\mathrm{g}-1}}{2}+\left(1-\frac{1}{\mathrm{P}}\right) \Phi_{\mathrm{g}-1}\right]
$$

ce qui conduit à :

$$
\begin{aligned}
\Phi_{g}=\frac{\beta}{2}\left(\frac{1}{2} \mathrm{~F}_{g-1}+\frac{1}{2} \mathrm{C}_{g-1}\right)+ & \frac{1}{4}\left(1-\frac{1}{\mathrm{R} f}\right) \mathrm{D}_{g-1} \\
& +\frac{1}{4 \mathrm{R} f}+\frac{1}{4} \frac{1}{\mathrm{R}} \frac{1+\mathrm{F}_{g-1}}{2}+\frac{1}{4}\left(1-\frac{1}{\mathrm{R}}\right) \Phi_{g-1}
\end{aligned}
$$


Les équations (3), (4), (5) et (6) constituent le système recherché qui peut s'écrire :

$\left[\begin{array}{l}\mathrm{F}_{g} \\ \Phi_{g} \\ \mathrm{C}_{g} \\ \mathrm{D}_{g}\end{array}\right]=\left[\begin{array}{l}0 \\ \frac{1}{4 \mathrm{R} f}+\frac{1}{8 \mathrm{R}} \\ \frac{1}{2 \mathrm{R}} \\ \frac{\beta^{2}}{2 \mathrm{P}}\end{array}\right]$

$$
+\left[\begin{array}{ccll}
\frac{\beta}{2} & 0 & \frac{\beta}{2} & 0 \\
\frac{\beta}{4}+\frac{1}{8 \mathrm{R}} & \frac{1}{4}\left(1-\frac{1}{\mathrm{R}}\right) & \frac{\beta}{4} & \frac{1}{4}\left(1-\frac{1}{\mathrm{R} f}\right) \\
\frac{1}{2 \mathrm{R}} & 1-\frac{1}{\mathrm{R}} & 0 & 0 \\
\frac{\beta^{2}}{2 \mathrm{P}} & \beta^{2}\left(1-\frac{1}{\mathrm{P}}\right) & 0 & 0
\end{array}\right]\left[\begin{array}{c}
\mathrm{F}_{\mathrm{g}-1} \\
\Phi_{\mathrm{g}-1} \\
\mathrm{C}_{\mathrm{g}-1} \\
\mathrm{D}_{\mathrm{g}-1}
\end{array}\right]
$$

Avant la sélection, nous supposerons la consanguinité et l'apparentement nul (population infinie) soit :

$\mathrm{F}_{0}=0$

$\Phi_{0}=0$

$C_{0}=0$
$D_{0}=0$$\quad$ puisqu'il n'y a pas sélection et que la population est infinie $\left(\frac{1}{R}=\frac{1}{P}=0\right)$.

Les valeurs limites de ces coefficients quand $g \rightarrow \infty$ s'obtiennent en posant $\mathrm{X}_{\mathrm{g}}=\mathrm{X}_{\mathrm{g}-1}$, ce qui donne :

$$
=\frac{\beta\left[\frac{4-\beta^{2}}{\mathrm{R}}+\frac{\beta^{2}}{\mathrm{P}}+\frac{1}{\mathrm{R} f}\left(2-\frac{2-\beta^{2}}{\mathrm{R}}-\frac{\beta^{2}}{\mathrm{P}}\right)\right]}{2(1-\beta)(3-\beta)(2+\beta)+\frac{4-2 \beta+\beta^{3}}{\mathrm{R}}+\frac{(4-3 \beta) \beta^{2}}{\mathrm{P}}+\frac{\beta^{2}}{\mathrm{R} f}\left[4-2 \beta-\frac{\beta}{\mathrm{R}}+\frac{3 \beta-4}{\mathrm{P}}\right]}
$$




$$
\begin{aligned}
& \Phi_{\infty}=\frac{\frac{2+\beta}{\mathbf{R}}+\frac{\beta^{2}(2-\beta)}{\mathbf{P}}+\frac{1}{\mathrm{R} f}\left[4-2 \beta-\frac{\beta}{\mathbf{R}}+\frac{\beta^{2}(\beta-2)}{\mathrm{P}}\right]}{2(1-\beta)(3-\beta)(2+\beta)+\frac{4-2 \beta+\beta^{3}}{\mathbf{R}}} \\
& +\frac{(4-3 \beta) \beta^{2}}{\mathrm{P}}+\frac{\beta^{2}}{\mathrm{R} f}\left[4-2 \beta-\frac{\beta}{\mathrm{R}}+\frac{3 \beta-4}{\mathrm{P}}\right]
\end{aligned}
$$
fermée;

Si $\beta=1$ on vérifie que $F_{\infty}=\Phi_{\infty}=1$, ce qui est logique pour une population

Si $\beta=0$, c'est-à-dire qu'à chaque génération les mâles sont supposés extérieurs à la population, les équations donnent :

$$
\begin{aligned}
& \mathrm{F}_{\infty}=0 \\
& \Phi_{\infty}=\frac{f+2}{2 f(3 \mathrm{R}+1)} \neq 0
\end{aligned}
$$

Dans ce cas précis, l'équation (2) se résoud simplement en

$$
\Phi_{\mathrm{g}}=\frac{f+2}{2 f(3 \mathrm{R}+1)} \quad 1-\left[\left(\frac{\mathrm{R}-1}{4 \mathrm{R}}\right)^{\mathrm{g}}\right]
$$

Ceci permet de constater que la convergence vers la limite est rapide.

Lorsque $0<\beta<1$, les coefficients $\mathrm{F}_{\infty}$ et $\Phi_{\infty}$ sont inférieurs à 1 . La figure 3 indique la variation de $\mathrm{F}_{\infty}$ en fonction des différents paramètres $\mathrm{R}, \alpha$ et $\beta, \alpha$ étant égal au rapport $\mathrm{P} / \mathrm{R}$ et $f$ prenant la valeur 6 .

\section{4. - Effectif génétique}

La vitesse de convergence vers les valeurs limites $F_{\infty}$ et $\Phi_{\infty}$ est donnée par la plus grande valeur propre de la matrice de passage (7). Ces valeurs propres sont solutions de l'équation caractéristique.

$$
\begin{aligned}
\lambda^{4}+\lambda^{3}\left(-\frac{\beta}{2}\right. & \left.-\frac{1}{4}+\frac{1}{4 \mathrm{R}}\right)-\lambda^{2}\left[\frac{\beta}{8}\left(1+\frac{1}{\mathrm{R}}\right)+\frac{\beta^{2}}{4}\left(1-\frac{1}{\mathrm{P}}\right)\left(1-\frac{1}{\mathrm{R} f}\right)\right] \\
+ & \lambda\left[\frac{\beta^{3}}{8}\left(1-\frac{1}{\mathrm{P}}\right)\left(1-\frac{1}{\mathrm{R} f}\right)\right]+\frac{\beta^{3}}{16}\left(\frac{1}{\mathrm{R}}-\frac{1}{\mathrm{P}}\right)\left(1-\frac{1}{\mathrm{R} f}\right)=0
\end{aligned}
$$

La figure 3 montre que pour une population ouverte $(\beta<1)$, la limite $F_{\infty}$ présente des valeurs faibles. De même les valeurs de $\mathcal{\lambda}$ maximum sont sensiblement inférieures à 1 , ce qui implique une convergence rapide vers les valeurs limites. 


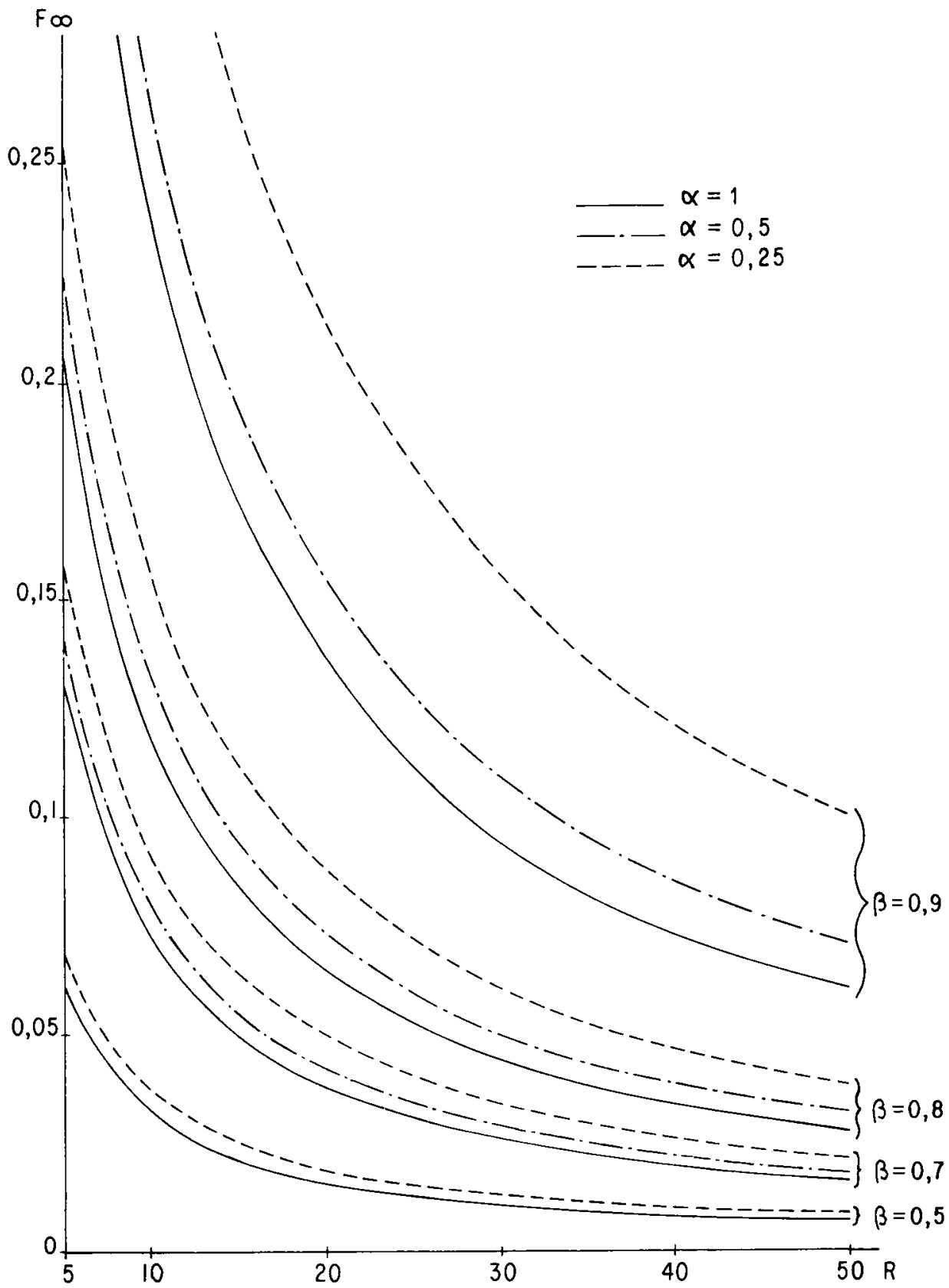

FIG. 3. - Relation entre le nombre de reines, $R$, sélectionnées à chaque génération et le coefficient de consanguinité limité, $F_{\infty}$, pour différentes valeurs des paramètres ir et $\beta$. FIG. 3. - Relation between the number of selected queens $(R)$ and the value of the maximum consanguinity coefficient $\left(F_{\infty}\right)$, for some values of the parameters $(\alpha)$ and $(\beta)$. 
Pour une population fermée $(\beta=1)$ dont les limites $\mathrm{F}_{\infty}$ et $\Phi_{\infty}$ sont égales à 1 , les valeurs propres de la matrice de passage sont voisines de $1, \frac{1}{4}, 0$ et $-\frac{1}{2}$ pour peu que $\mathrm{P}$ et $\mathrm{R}$ soient assez grands. On peut donc définir dans ce cas un effectif génétique, $\mathrm{N}_{e}$, caractérisant la vitesse de la convergence de $\mathrm{F}_{\mathrm{g}}$ et $\Phi_{g}$ vers 1 , selon la formule :

$$
\lim _{g \rightarrow \infty} \frac{\mathrm{F}_{g}-\mathrm{F}_{\mathrm{g}-1}}{1-\mathrm{F}_{\mathrm{g}-1}}=\lim _{g \rightarrow \infty} \frac{\Phi_{g}-\Phi_{g-1}}{1-\Phi_{g-1}}=\frac{1}{2 N_{e}}
$$

Cet effectif génétique est liè à la plus grande racine $\lambda_{\mathrm{M}}$ de l'équation (11) par :

$$
\lambda_{\mathrm{M}}=1-\frac{1}{2 \mathrm{~N}_{e}}
$$

En reportant cette expression dans l'équation (11), avec $\beta=1$, et en supposant $\mathrm{R}, \mathrm{P}$ et $\mathrm{N}_{e}$ assez grands, la résolution conduit à la formule approchée suivante :

$$
\frac{1}{\mathrm{~N}_{e}} \simeq \frac{1}{9 \mathrm{P}}+\frac{3 f+2}{9 \mathrm{R} f}
$$

L'erreur relative devient rapidement négligeable dès que $P$ et $R$ sont supérieurs à 10 , la valeur exacte de l'effectif génétique étant toujours supérieure à sa valeur approchée et la différence n'excédant jamais l'unité.

\section{II. - DISCUSSION}

La figure 3 indique que la consanguinité moyenne reste à un niveau très faible, même avec très peu de géniteurs, tant que $\beta$ ne dépasse pas 0,7 . Dans ce cas $(\beta<0,7)$ en outre, la proportion $\alpha$ ''a pratiquement pas d'influence, tout au moins quand $\alpha$ est compris entre 0,25 et 1 . Par contre, plus $\beta$ se rapproche de 1 et plus les courbes s'écartent pour différentes valeurs de $\alpha$, l'écart s'accentuant quand $\alpha$ diminue.

D'une manière générale, cette étude montre que, pour le plan de sélection considéré, la consanguinité ne deviendrait un obstacle majeur que si le rucher de fécondation était parfaitement isolé.

Pour rester en dessous de 10 p. 100 de consanguinité, il suffit de prendre 10 reines souches à chaque génération, pour $\beta \leqslant 0,7$. En revanche, avec $\beta=0,8$ il en faut au moins 15 et pour $\beta=0,9$ il en faut plus de 30 .

Ces résultats ont été obtenus en prenant une valeur arbitraire de $f$ égale à 6 . En réalité, il semble que cette valeur représente une limite inférieure pour ce paramètre (ADAMs et al., 1977). Or nous avons vérifié, pour toutes les valeurs des paramètres envisagées sur la figure 3, que la dérivée de la fonction $\mathrm{F}_{\infty}(f)$ était négative. Il en résulte que, si 6 est une borne inférieure de $f, \mathrm{~F}_{\infty}(6)$ valeur indiquée sur la figure 3 , sera une borne supérieure de $F_{\infty}$. 
Lorsque la population est fermée $(\beta=1)$, nous avons établi une formule approchée de l'effectif génétique que nous pouvons comparer à celle de WRIGHT (1933) reprise par KERR (1967) sous la forme :

$$
\frac{1}{N_{e}}=\frac{4}{9 N_{F}}+\frac{2}{9 N_{M}}
$$

avec : $N_{F}$ : nombre de reines;

$\mathrm{N}_{M}$ : nombre de mâles ayant inséminé ces reines.

Ces auteurs se sont placés dans l'hypothèse d'une population non soumise à la sélection artificielle. Dans ces conditions, $P$ et $R$ sont égaux à $N_{F}$. En effectuant cette transformation dans (12) et en remplaçant $\mathrm{N}_{\mathrm{M}}$ par $f \mathrm{~N}_{\mathrm{F}}$ dans (13) on constate que ces deux formules coïncident puisqu'elles s'écrivent toures deux :

$$
\frac{1}{\mathrm{~N}_{e}}=\frac{4 f+2}{9 f \mathrm{~N}_{\mathrm{F}}}
$$

Reçu pour publication en juin 1981. Eingegangen im Juni 1981.

\title{
ZUSAMMENFASSUNG
}

\author{
ENTWICKLUNG DES INZUCHTGRADES IN EINER BIENENPOPULATION \\ UNTER DEM EINFLUSS DER SELEKTION
}

Es wird eine Population betrachtet, in welcher sich die Königinnen natürlich mit einer mittleren Zahl $f$ Drohnen paaren. In jeder Generation werden R Königinnen selektioniert, um die Königinnen der nächsten Generation zu erzeugen. Es wird angenommen, dass die Drohnen zu einem Anteil $\beta$ von den selektionierten Königinnen abstammen und zu einem Anteil $(1-\beta)$ von fremden Drohnen.

Der mittlere Inzuchtkoeffizient $\mathrm{F}$ wird als Funktion der Generation und der Parameter R, P, $\beta$ und $f$ bestimmt. Für offene Populationen $(\beta<1)$ wird der Ausdruck des Maximalwertes dieses Koeffizienten $\left(F_{\infty}\right)$ mit seinen Variationskurven für die verschiedenen möglichen Werte der Parameter angegeben.

Auf diese Weise kann gezeigt werden, dass eine Immigrationsrate von $20-30 \%$ ausreicht, um bei Verwendung von 10-15 selektionierten Königinnen pro Generation das Niveau der Inzucht unter $10 \% \mathrm{zu}$ halten.

Ist die Population geschlossen $(\beta=1)$, ergibt sich die effektive Populationsgrösse aus der Formel :

$$
\frac{1}{\mathrm{~N}_{e}}=\frac{1}{9 \overline{\mathrm{P}}}+\frac{3 f+2}{9 \mathrm{R} f}
$$




\section{SUMMARY \\ CHANGE WITH TIME OF CONSANGUINITY \\ IN A HONEYBEE POPULATION}

We consider a population in which queens naturally mate with an average number, $f$, of drones. Every new generation of queens is issued from $\mathrm{R}$ queens selected among the previous one. It is assumed that the drones, in a $\beta$ proportion, are born of $\mathrm{P}$ selected queens and in a $(1-\beta)$ proportion. of external colonies.

The mean consanguinity coefficient $(F)$ is given as a function of the generation and of the parameters $\mathrm{R}, \mathrm{P}, \beta$, and $f$. When the population is not closed $(\beta<1)$, the expression of the maximum value of this coefficient $\left(F_{\infty}\right)$ is given with variation curves in relation to various values of parameters. Thus, it can be shown that an immigration rate of $20 \%$ to $30 \%$ is enough to maintain the level of consanguinity under $10 \%$, with only 10 to 15 selected queens per generation.

If the population is closed $(\beta=1)$, the effective population number $\mathrm{N}_{\mathrm{e}}$ is given by the formula :

$$
\frac{1}{\mathrm{~N}_{e}}=\frac{1}{9 \mathrm{P}}+\frac{3 f+2}{9 \mathrm{R} f}
$$

\section{BIBLIOGR APHIE}

Adams J., Rothman E. D., Kerr W. E. et Paulino Z. L., 1977. - Estimation of the number of sex alleles and queen matings from diploid male frequencies in a population of Apis mellifera. Genetics, 86, 583 596.

Chevalet Cl. et Cornuet J.-M., 1982. - Étude théorique sur la sélection du caractère " production de miel " chez l'Abeille. I - Modèle génétique et statistique. Apidologie, 13 (1), 39-65.

Cornuet J.-M. et Chevalet Cl., 1982. - Étude théorique sur la sélection du caractère " production de miel " chez l'A beille. II - Plan de sélection combinée de reines en fécondation naturelle. Apidologie, 13 (2).

Crow J. F. et Kimura M., 1970. - An introduction to population genetics theory. Harper and Row., New York, $591 \mathrm{p}$.

KERR W. E., 1967. - Multiple alleles and genetic load in bees, J. apic. Res., 6, 3-9.

Malecor G., 1948. - Les mathématiques de l'hérédité. Masson et Cie, Paris.

Plass F., 1953. - Inzuchtwirkung und Heterosiseffekt bei der Honigbiene. Schriftenreihe des AID (Godesberg), 66, 49-68.

Wоуке J., 1972. - Les allèles sexuels et la fécondité contrôlée. - Symposium international Lunz am See, 69-74.

WRIGHT S., 1933. - Inbreeding and homozygosis. - Proc. Nat. Acad. Sci., U.S.A., 19, 411-420. 